\title{
Mechanical Thrombectomy for Treatment of Cerebral Venous Sinus Thrombosis in Vaccine-Induced Immune Thrombotic Thrombocytopenia
}

\author{
(D) H.S. Chew, (DS. Al-Ali, (D)B. Butler, (D) D. Rajapakse, (D). Nader, (DS. Chavda, and (DS. Lamin
}

\begin{abstract}
SUMMARY: Reports of a rare form of cerebral venous sinus thrombosis with profound thrombocytopenia have emerged following introduction of the adenovirus-vectored coronavirus disease 2019 (COVID-19) vaccines. Between March and June 2021, seven cases of refractory vaccine-induced immune thrombotic thrombocytopenia were referred to our institution for mechanical thrombectomy. The condition of 1 patient deteriorated during interhospital transfer, and the remaining 6 underwent successful recanalization. No procedure-related adverse events were reported. At the time of this writing, 3 patients have been discharged with a good functional outcome (mRS 0-1), one required rehabilitation for mild dysarthria and vocal cord palsy (mRS 3), and 2 have died due to severe mass effect. Our anecdotal experience suggests that endovascular therapy may be safe and effective in reducing thrombus burden in selected cases of postvaccination cerebral venous sinus thrombosis.
\end{abstract}

ABBREVIATIONS: COVID-19 = coronavirus disease 2019; CVST = cerebral venous sinus thrombosis; HIT = heparin-induced thrombocytopenia; ICH = intracerebral hemorrhage; $\mathrm{PF}=$ platelet factor; VITT = vaccine-induced immune thrombotic thrombocytopenia

V accine-induced immune thrombotic thrombocytopenia (VITT), also known as thrombosis with thrombocytopenia syndrome, is a rare disorder of immune-driven thrombosis and thrombocytopenia linked to coronavirus disease 2019 (COVID19) vaccines, which use adenoviral vectors to deliver the spike protein-producing genes. Initially reported after the AstraZeneca COVID-19 vaccination in Europe, similar cases also occurred after receipt of Janssen/Johnson \& Johnson COVID-19 vaccines in the United States. ${ }^{1}$ The condition shares a mechanism similar to that of spontaneous heparin-induced thrombocytopenia (HIT), without heparin exposure as a trigger. HIT is a highly prothrombotic condition that develops 4-10 days after heparin exposure, leading to widespread platelet activation triggered by formation of antibodies against the platelet factor (PF)-4 complex. As with HIT, individuals with VITT also produce similar antibodies that bind to platelets via the Fc $\gamma$ RIIA receptors. It remains unclear which component of the vaccine is responsible for the production of anti-PF-4 antibodies. ${ }^{2}$

Received July 23, 2021; accepted after revision September 30

From the Department of Neuroradiology, Queen Elizabeth Hospital Birmingham, Birmingham, UK.

Please address correspondence to Han Seng Chew, MD, Department of Neuroradiology, Queen Elizabeth Hospital Birmingham, Mindelsohn Way, Edgbaston, B15 2GW Birmingham, UK; e-mail: hanseng.chew@uhb.nhs.uk

- Indicates open access to non-subscribers at www.ajnr.org

Indicates article with online supplemental data.

http://dx.doi.org/10.3174/ajnr.A7381
As of June 30, 2021, the UK regulators received 399 reports of major thromboembolic events following AstraZeneca COVID-19 vaccination. There is a high predilection for cerebral venous sinus thrombosis (CVST), affecting more than one-third of reported cases $(142,35.6 \%)$, with a higher incidence in younger adult groups (average, 46 years of age). ${ }^{3}$ Review of recent cases has prompted the Joint Committee on Vaccination and Immunisation to recommend that those younger than 40 years of age in the UK be offered an alternative COVID- 19 vaccine. ${ }^{4}$

There are no robust clinical data at the time of this writing to inform treatment of VITT. The British Society for Haematology issued guidance based on the experience of managing initial cases and alternative similar conditions. Estimates to date suggest that patients who develop CVST are more likely to have a fatal outcome with conventional treatment. ${ }^{5}$ The aim of this case series was to report our experience with mechanical thrombectomy in the management of severe postvaccination CVST.

\section{MATERIALS AND METHODS}

Seven confirmed cases of VITT presenting with refractory CVST were referred to our institution for consideration of endovascular treatment between March 16 and June 10, 2021. Symptom onset ranged between 10 and 14 days after receiving at least 1 dose of the AstraZeneca vaccine. Systemic anticoagulation and immune-modulation therapy had been initiated before patients were accepted for intervention. Of the 6 patients with intracranial bleeds, 5 presented with reduced Glasgow Coma Score and required intubation 

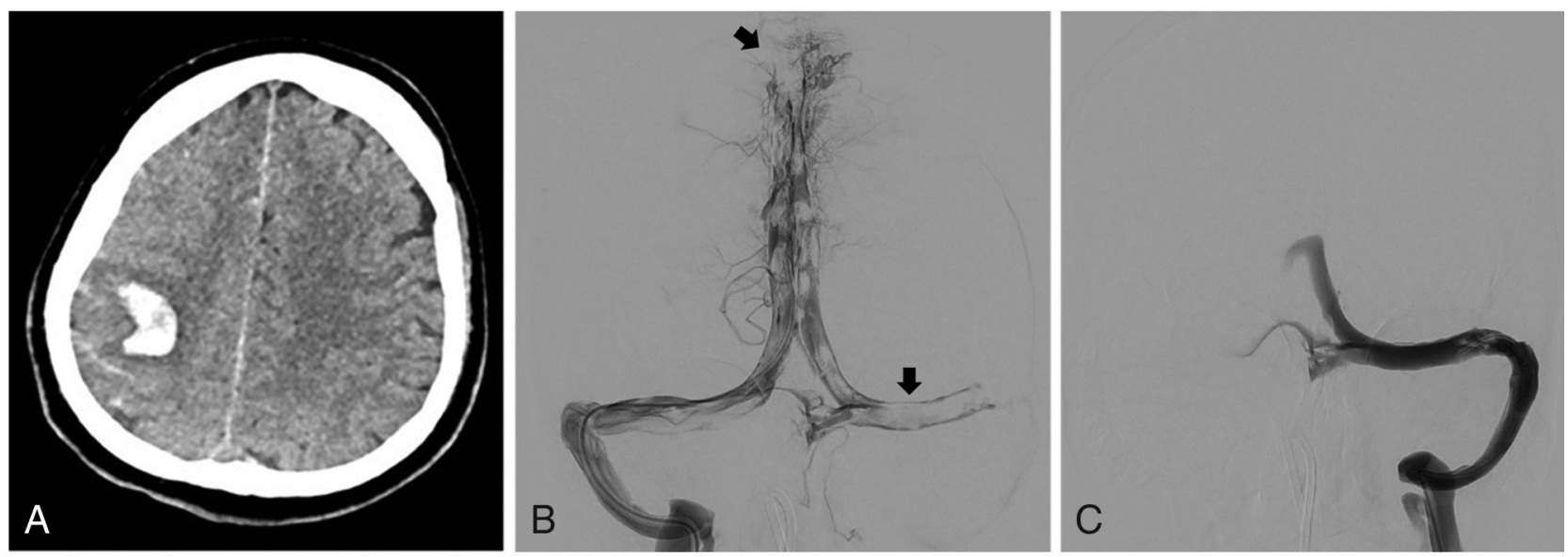

FIGURE. A, Axial CT of the head of patient 1 demonstrates focal ICH in the right frontal lobe with acute sulcal blood. B, Anterior-posterior view of a selective injection confirms extensive filling defects in the superior sagittal, left transverse, and sigmoid sinus. $C$, Complete recanalization of the left transverse and sigmoid sinuses after the final pass.

before interhospital transfer. The only patient without intracerebral hemorrhage $(\mathrm{ICH})$ was accepted for treatment on the basis of evidence of clot extension on serial imaging.

\section{Technical Details}

Venous access was obtained by inserting an $8 \mathrm{~F}$ short sheath into the right femoral vein, followed by navigating a $90-\mathrm{cm}$ Neuron Max long sheath (Penumbra) to the sigmoid sinus. A large-bore reperfusion catheter was advanced into the superior sagittal sinus over a Velocity microcatheter (Penumbra). Clot extraction was performed with a 6-mm-diameter stent retriever along with concurrent suction through the reperfusion catheter at the clot face. The process was repeated until successful recanalization of the venous sinus was achieved (Figure).

The procedure was performed without reversing the anticoagulation therapy. Nearly all our patients had platelet counts lower than the safe threshold recommended for high-risk interventional procedures $\left(50 \times 10^{9} / \mathrm{L}\right){ }^{6}$ Several precautionary measures were taken to minimize the risk of hemorrhagic complications. The intracatheter heparin flush was switched off when the catheter system engaged the thrombus to keep the total amount of heparin infusion to a minimum. Selective ICA injection to assess cerebral capillary filling and venous drainage was not performed because this would require an additional arterial puncture. For the first 2 cases, the femoral short sheath was sutured to the skin and removed at the bedside 24 hours later to avoid groin hemorrhage. In subsequent cases, the short sheath was replaced with a Vascath catheter to facilitate plasma exchange therapy. Intrasinus thrombolysis was avoided due to the uncertain effect of thrombolytic agents in the presence of platelet-activating antibodies.

\section{RESULTS}

The mean age of patients was 40 years (range, $26-49$ years). The male/female ratio was $4: 3$. The median interval from vaccination to admission was 12 days (range, 10-14 days). One female patient was on oral contraceptives. No other thrombotic risk factors were identified. Five patients (71\%) had reduced Glasgow Coma Score at the time of referral, and three (43\%) presented with mass effect and midline shift on imaging before endovascular therapy. Six of 7 patients were found to have severe thrombocytopenia $(<50$ $\times 10^{9} / \mathrm{L}$ ) on admission (range, $11-91 \times 10^{9} / \mathrm{L}$ ). Two patients with platelet counts lower than $20 \times 10^{9} / \mathrm{L}$ developed a purpuric skin rash and hematuria resembling immune thrombocytopenic purpura.

The condition of 1 patient deteriorated during interhospital transfer despite neuroprotective measures. Repeat CT imaging on arrival showed global cerebral edema far too advanced for any interventional procedures. The remaining 6 patients underwent successful endovascular therapies. Thrombectomy was performed with the patient under general anesthesia in 4 patients and local anesthesia with conscious sedation in the other 2 . The mean procedural time was 100 minutes (range, $80-145$ minutes). On average, 5.7 passes were made per patient (range, 4-7 passes).

\section{Treatment Outcome}

Satisfactory recanalization was achieved in all except 1 patient in whom the posterior segment of the superior sagittal sinus showed partial recanalization at the end of the procedure. No procedurerelated adverse events were recorded. Two patients underwent decompressive craniectomy following endovascular treatment. In the first case, mechanical thrombectomy and decompressive craniectomy were performed in the same session because the head CT on admission demonstrated severe mass effect with midline shift. The second patient developed progressive lobar ICH and worsening mass effect, despite successful clot retrieval. Decompressive craniectomy was performed within 12 hours post-endovascular treatment. At the time of this writing, 3 (50\%) patients were discharged with a good functional outcome (mRS 0-1), one (16.6\%) required rehabilitation for mild dysphasia and vocal cord palsy leading to swallowing difficulty (mRS 3), and two (33.3\%) died due to severe mass effect. Both patients who died following thrombectomy showed ICH with a midline shift on the initial head CT.

No reclotting of the superior sagittal sinus was identified on follow-up imaging. Recurrent transverse and sigmoid sinus 
thromboses were confirmed in 2 patients. In the first case, the thrombus completely resolved on subsequent imaging. The second patient received prophylactic platelet transfusion before decompressive craniectomy and developed refractory thrombocytopenia warranting multiple sessions of plasma-exchange therapies. Repeat CT venography showed absent venous flow in the left transverse sinus and sigmoid sinus without further clot extension. Both patients made a good recovery.

\section{DISCUSSION}

VITT is a newly described syndrome characterized by thrombosis, thrombocytopenia, and markedly raised serum D-dimer levels. Symptom onset ranges from 5 to 30 days after initial exposure to adenovirus-vectored vaccines. ${ }^{5,7}$ On the basis of the current UK data, the condition appears to affect patients of all ages and both sexes, with no clear signal of risk factors. Most developed CVST after receiving the first dose of the AstraZeneca vaccine; however, a small number of cases occurred after the second dose. Typical laboratory features included a platelet count of $<150 \times 10^{9} / \mathrm{L}$, D-dimer levels of $>4000 \mathrm{mcg} / \mathrm{L}$, and low serum fibrinogen levels. Positive findings on the PF-4 antibody enzyme-linked immunosorbent HIT assay are required to confirm the diagnosis.

There is no evidence-based advice on the treatment of postvaccine CVST due to the novelty of the vaccines. The Expert Haematology Panel recommends commencing intravenous immunoglobulin and systemic anticoagulation with non-heparinbased anticoagulants in all suspected cases while awaiting a confirmatory diagnosis. Because the syndrome mimics HIT, all forms of heparin, including heparinized saline flushes, are best avoided until further evidence becomes available. Patients on treatment should have serial platelet counts to monitor disease activity and treatment response. Early use of plasma exchange is recommended in those with platelet counts lower than $30 \times 10^{9} / \mathrm{L}$, who often have high levels of circulating anti-PF-4 antibodies. Repeat plasma exchange may be required if platelet recovery is slow.

Both arterial and venous thromboses at multiple organ sites have been reported. However, a significant proportion of patients with VITT developed thrombosis in the cerebral venous sinus system. This group of patients is more likely to have a poor outcome, with a tendency toward rapid progression to life-threatening hemorrhage and relevated intracranial pressure. Of 213 cases of postvaccination CVST reported to the European Medicines Agency, ${ }^{8} 49 \%$ of patients in the AstraZeneca group who developed refractory CVST died compared with a $13 \%$ death rate in standard CVST. ${ }^{9}$ A web-based survey involving all departments of neurology in Germany reported an $18.3 \%$ mortality rate among patients with CVST within 1 month from the administration of the first dose of COVID-19 vaccines. ${ }^{10}$ In the US series, ICH occurred $<48$ hours after admission, and the median time from admission to death was 1 day. ${ }^{1}$ Similar observations can be made in our cohort: All fatal cases developed intraparenchymal hemorrhage in $<12$ hours after the first head CT with normal findings and died within 48 hours after hospitalization.

The mechanism responsible for rapid deterioration following the onset of $\mathrm{ICH}$ is not fully understood. This may be partly attributed to severe thrombocytopenia associated with VITT. A recently published prospective study involving 294 patients in the UK identified the baseline platelet count and the presence of ICH as being independently associated with death. ${ }^{11}$ A mortality rate of $73 \%$ was observed among those who presented with platelet counts below $30 \times 10^{9} / \mathrm{L}$ and $\mathrm{ICH}$. The risk/benefit ratio of anticoagulation therapy needs to be carefully balanced in patients with severe thrombocytopenia. Switching to a low-dose anticoagulant may be appropriate when the platelet count is lower than $30 \times 10^{9} / \mathrm{L}$. Platelet transfusion for correction of thrombocytopenia is likely to fuel further thrombosis and, therefore, should only be considered in life-threatening hemorrhage. The location of the thrombosis also appears to have a significant impact on patient outcomes. In our series, 3 of 4 patients who presented with complete occlusion of the superior sagittal sinus had bilateral ICH leading to fatal outcomes.

Decompressive craniectomy may be life-saving in the presence of a raised ICP and impending brain herniation. ${ }^{12}$ In cases in which neurosurgical intervention is indicated, prophylactic platelet transfusion and reversal of systemic anticoagulation are essential in preventing a catastrophic perioperative bleed. ${ }^{13}$ Patients should be monitored closely for signs of clot propagation following decompressive surgery.

\section{Role of Mechanical Thrombectomy}

Because most published data come from small case series, there is limited knowledge about the appropriate indications and timing of endovascular treatment in standard CVST. A systemic review of 185 cases of mechanical thrombectomy published in 2015 showed that $60 \%$ of patients had a pretreatment ICH and $40 \%$ were comatose. ${ }^{9}$ A recently published multicenter, randomized blind-ended trial involving 67 patients between 2011 and 2016 reported that mechanical thrombectomy with standard medical care did not improve functional outcome in patients with severe CVST. ${ }^{14}$ The investigators, however, acknowledged that future studies using different methods of patient selection and endovascular techniques may identify better patient outcomes. In the absence of convincing evidence, endovascular intervention is largely reserved for severe CVST not responding to conventional therapy. ${ }^{15}$

Nearly all patients in our series had developed an intracranial bleed by the time referrals were made. Within 48 hours after hospitalization, 3 died due to severe mass effect, including the one whose condition deteriorated during interhospital transfer. The sample size of this case series is too small to determine whether endovascular therapy has a positive effect on the treatment outcome of refractory CVST. Given the high probability of rapid progression to life-threatening $\mathrm{ICH}$, we recommend transferring patients presenting with heavy clot burden and severe thrombocytopenia to a tertiary center for consideration of early plasmaexchange therapy and endovascular treatment when there is evidence of clinical or radiologic deterioration. For those who are clinically stable on admission, judicious use of CT/MR venography may detect silent clot propagation before the collateral pathways become overwhelmed.

Our anecdotal experience suggests that mechanical thrombectomy can be safely performed without prophylactic platelet transfusion and reversal of anticoagulation treatment. Recent 
advancements in catheter-tracking technologies and stent retriever designs improve the rate of recanalization in patients with acute stroke. New-generation stent retrievers tend to be less stiff compared with older devices (eg, AngioJet; Boston Scientific), allowing the operator to complete procedures with higher degrees of recanalization within shorter timeframes. Most commercially available stent retrievers and reperfusion catheters are designed for arterial thrombectomy. Purpose-built devices with larger diameters to match the caliber of venous sinus systems may improve the recanalization rate at challenging locations such as sigmoid sinuses and jugular bulbs.

\section{CONCLUSIONS}

Management of VITT-induced CVST requires multidisciplinary care with joint input from hematology, neurology, neuroradiology, and neurosurgery. These patients are best managed at neuroscience centers with access to neurointerventional and neurosurgical expertise. There is currently insufficient data to assess the efficacy of endovascular treatment in vaccine-induced CVST due to the rarity of the condition. Mechanical thrombectomy is effective in achieving a rapid reduction in clot burden while awaiting immune-modulation therapy to neutralize platelet-activating antibodies. As new evidence emerges, treatment recommendations are expected to change. Our anecdotal experience suggests that mechanical thrombectomy is a relatively safe treatment technique with a high degree of angiographic recanalization. When appropriate expertise is available, early mechanical thrombectomy can be considered in patients with VITT with severe thrombocytopenia and heavy clot burden to alleviate raised intracranial pressure.

Disclosure forms provided by the authors are available with the full text and PDF of this article at www.ajnr.org.

\section{REFERENCES}

1. See I, Su JR, Lale A, et al. US case reports of cerebral venous sinus thrombosis with thrombocytopenia after Ad26.COV2.S vaccination, March 2 to April 21, 2021. JAMA 2021;325:2448-56 CrossRef Medline

2. Makris M, Pavord S, Lester W, et al. Vaccine-induced immune thrombocytopenia and thrombosis (VITT). Res Pract Thromb Haemost 2021;5:e12529 CrossRef Medline

3. Coronavirus vaccine: weekly summary of Yellow Card reporting: Updated 26 November 2021. https://www.gov.uk/ government/publications/coronavirus-covid-19-vaccine-adversereactions/coronavirus-vaccine-summary-of-yellow-card-reporting. Accessed June 1, 2021

4. JCVI advises on COVID-19 vaccine for people aged under 40. https:// www.gov.uk/government/news/jcvi-advises-on-covid-19-vaccine-forpeople-aged-under-40. Accessed June 1, 2021

5. Guidance from the Expert Haematology Panel (EHP) on Covid-19 Vaccine-induced Immune Thrombocytopenia and Thrombosis (VITT): 28 May 2021. https://b-s-h.org.uk/media/19718/guidancev20-20210528-002.pdf. Accessed June 1, 2021

6. Patel IJ, Davidson JC, Nikolic B, et al; Standards of Practice Committee, with Cardiovascular and Interventional Radiological Society of Europe (CIRSE) Endorsement. Consensus guidelines for periprocedural management of coagulation status and hemostasis risk in percutaneous image-guided interventions. J Vasc Interv Radiol 2012;23:727-36 CrossRef Medline

7. Greinacher A, Thiele T, Warkentin TE, et al. Thrombotic thrombocytopenia after ChAdOx1 nCov-19 vaccination. $N$ Engl J Med 2021;384:2092-101 CrossRef Medline

8. Krzywicka K, Heldner MR, Sánchez van Kammen M, et al. PostSARS-CoV-2-vaccination cerebral venous sinus thrombosis: an analysis of cases notified to the European Medicines Agency. Eur J Neurol 2021;28:3656-62 CrossRef Medline

9. Siddiqui FM, Dandapat S, Banerjee C, et al. Mechanical thrombectomy in cerebral venous thrombosis: systematic review of 185 cases. Stroke 2015;46:1263-68 CrossRef Medline

10. Schulz JB, Berlit P, Diener HC, et al. German Society of Neurology SARS-CoV-2 Vaccination Study Group. COVID-19 vaccine-associated cerebral venous thrombosis in Germany. Ann Neurol 2021;90:6273-39 CrossRef Medline

11. Pavord S, Scully M, Hunt BJ, et al. Clinical features of vaccineinduced immune thrombocytopenia and thrombosis. $N$ Engl J Med 2021;385:1680-89 CrossRef Medline

12. Ferro JM, Bousser MG, Canhão $P$, et al; European Stroke Organization. European Stroke Organization guideline for the diagnosis and treatment of cerebral venous thrombosis: endorsed by the European Academy of Neurology. Eur J Neurol 2017;24:1203-13 CrossRef Medline

13. Management of cerebral venous sinus thrombosis following Covid19 vaccination: a neurosurgical guide. https://www.rcseng.ac.uk/-/ media/files/rcs/coronavirus/cvstguide_--1942020.pdf. Accessed June 1,2021

14. Coutinho JM, Zuurbier SM, Bousser M, et al; TO-ACT investigators. Effect of endovascular treatment with medical management vs standard care on severe cerebral venous thrombosis: the TO-ACT Randomized Clinical Trial. JAMA Neurol 2020;77:966-73 CrossRef Medline

15. Ilyas A, Chen C, Raper DM, et al. Endovascular mechanical thrombectomy for cerebral venous sinus thrombosis: a systematic review. J Neurointerv Surg 2017;9:1086-92 CrossRef Medline 